\title{
Planck Constant as Adiabatic Invariant Characterized by Hubble's and Cosmological Constants
}

\author{
Anton Lipovka \\ Department of Investigation for Physics, Sonora State University, Hermosillo, Sonora, Mexico \\ Email: aal@cifus.uson.mx
}

Received January 2014

\begin{abstract}
Within the framework of Einstein-Cartan-Shrödinger formalism with asymmetric connections, the Planck constant is calculated from the first principles (from geometry of our Universe), as the adiabatic invariant of electromagnetic field on the Riemann-Cartan manifold. The Planck constant, calculated with actually measured cosmological parameters, coincide with that one, measured in laboratory with precision up to the second digit. The non-local generalization of quantum theory is suggested. The fundamental sense of the Quantum Theory is discussed, and physical sense of the cosmological constant is revealed. Within the mentioned framework, the quantum theory is naturally unified with gravity.
\end{abstract}

\section{Keywords}

\section{Cosmology, Quantum Theory, Unified Theory}

\section{Introduction}

Quantum Theory (In accordance with the historical terminology, we shall call "Quantum Theory" (QT) the theory, based on the concept of wave functions, or probability amplitudes), that recently has celebrated its 100-year anniversary, allowed at the time to overcome a crisis that happened in atomic physics, giving researchers a necessary tool for the calculation of atomic and subatomic phenomena with an accuracy which is in striking agreement with experiment. However, since its foundation and up to now, physicists and mathematicians are still attempting to interpret this unusual QT formalism. On the one hand Quantum Mechanics (QM) from the beginning (and then Quantum Field Theory as its successor) was built on the axiomatic approach, which cannot be considered as satisfactory. So, the concept of the wave function was postulated for all describable entities. On the other hand, the evolution operator for a system is linear with the wave function, whereas its square appears as the result of the measurement process. If we add to the above the presence of divergences and unrenormalizability of theories in general case, the serious problems with unification of QT and general relativity (GR), and inability to obtain the mass and charge from the first principles, the incompleteness of QT becomes apparent, and thus there is need to find a complete theory describing the atomic and nuclear systems. 
Since the moment of discovery, QT did not please its creators, giving rise to numerous discussions about the place for probability in physics, the wave-particle duality, discussion of thought experiments and paradoxes. We shall not discuss here again the well-known history of QT, for that the reader should refer to the monograph [1]. With such an "unusual” physics, researchers put up nearly a century, excusing its numerous defects, because QT allows calculate physically interesting phenomena in excellent agreement with the experiment. The situation began to change in the last decade of the 20th century, when the crisis that hit theoretical physics became obvious to many physicists and people started talking loudly about the problems that arise when trying to unify QT and GR. Among the most serious problems of the Standard Model we could mention the following:

- The problem of the collapse of the wave function (the problem of the observer, or Einstein-Podolsky-Rosen paradox).

- The presence of unrenormalizable (in general) divergences.

- The huge discrepancy between the calculated with QFT methods and observed cosmological constant.

- Conflict of QT with GR at the horizon of black holes.

- Recent experimental data obtained with the Planck satellite, which disfavors all the best-motivated inflationary scenarios [2].

- Inability of a reasonable harmonization or unification of the standard model with gravity.

This incomplete list of problems indicates very serious gaps in our understanding of Nature. For the most cases, the problems are directly or implicitly appear from a misunderstanding of the basis of the quantum theory, and the nature of its main concepts and axioms. The present paper is urged to fill the above mentioned gap and to specify a way free from the difficulties listed above. We begin with a generalization of the quantum theory because in its present form it cannot be unified with General relativity. In second part of the paper (Sections 5, 6 and 7) we show how the QT and relativity should be unified and calculate the Planck constant (fundamental parameter of QT) from the geometry of our Universe, i.e. from the Hubble and cosmological constants.

\section{Quantization}

It is well known that quantum mechanics appeared from the need to explain the experimentally observed blackbody emission spectrum and atomic spectra. Planck was the first who propose an analytical formula to describe the spectral energy distribution which was consistent with the experiment. However, as it was noted by Einstein [3], the way in which Planck obtained his result, was not quite correct, though it led to the correct result. The problem was that Planck included in his formula not only the electromagnetic field, but also oscillators associated with the matter. As a result, in the electrodynamic part, based on Maxwell's equations, the energy of the oscillators was a continuously varying value, while in the statistical part the same energy was considered as a discrete value (quantized).

In 1905 Einstein published the work [4] in which he showed that the emission field (without any assumptions on the nature of matter) behaves so as if consists of separate quanta (photons), characterized by energy $h v$. Later, in 1910 Debye [5] argued that Planck's formula can be deduced for the pure radiation field, absolutely without any assumptions on the oscillator's properties of the substance. Thus Planck's law and all its consequences, follows from the fact that the energy of freely propagating electromagnetic field is divided by parts proportional to $h v$. Recently this result was confirmed experimentally [6]. This fact is the only we need to obtain the Planck constant from the geometry of our Universe, so reader interested in the relation between the Planck constant and local curvature of our Universe (Cosmological constant and Hubble constant) is referred directly to the chapter “Adiabatic invariant” of present paper. But now we consider consequences for the QT, which follows from the mentioned above properties of the electromagnetic field.

It is known that the Bohr-Sommerfeld theory (so-called old quantum theory), based on the adiabatic hypothesis, is founded on two quantum axioms, which when added to the axioms of classical mechanics allow us to build a quantum theory. These two axioms are written as:

$$
\begin{aligned}
& \oint p_{k} d q_{k}=n_{k} h, \\
& E_{1}-E_{2}=h v,
\end{aligned}
$$

The hypothesis expressed by Sommerfeld (that action for any elemental process is changed by value $h$ ) served as the basis to write these relations. It states that in each elementary process, the action of the atom changes by 
an amount equal to the Planck constant. However, if we take into account the results obtained by Einstein and Debye, we easily receive these postulates, as a consequence of classical mechanics, i.e. we can construct the reasonable classical theory of emission/absorption in lines, and the classical atomic theory without recurring to the concept (axiom) of the wave function and the problems provoked by last one. It should be stressed here, that so-called "new quantum theory" also is based on the axiom, and this axiom (of the wave functions existence) cannot be explained or reduced to real physics, whereas the Bohr-Sommerfeld axioms can be reduced to (or obtained from) classical physics, which provide us with a fundamental view to the basic concept and understanding the nature of QT. To achieve the above, it should be noted, that there are only two fields which are carrying out interactions at distances we are interested in $\left(r>10^{-11} \mathrm{~cm}\right)$. These are the electromagnetic and the gravitational fields. Considering that the interaction constant for a gravitational field is negligible in comparison with the electromagnetic one, we can surely approve the following: Everything that we see, feel, hear, measure, register with detectors, is the electromagnetic field and nothing else. That is we perceive the real world in the form of this picture, by means of electromagnetic waves registered by us. It is important to understand, that the electromagnetic field acts as intermediary between the observer and the real (micro) world, hiding from us reality (idea of existence of the so-called hidden variables or beables in QM). In our case these hidden variables lose the mystical meaning, becoming usual classical variables - coordinates and momenta of particles, but which can be measured only by the electromagnetic field means.

Thus as a starting point we propose the following:

- The electromagnetic field is the only field responsible for interaction between objects and observer in Quantum mechanics.

- The electromagnetic field is quantized without the need of any assumptions about the properties of oscillators. That is the Planck's relation of $E=h v, P=\hbar k$ is satisfied, irrespective of the oscillators properties (see [4]-[6]).

The last thesis means that there exists (and therefore can be emitted) only the photon possessing the period $2 \pi$. In other words, emission/absorption of a photon can occur only for the whole period of movement of a charge (in system of coordinates in which proceed the emission/absorption).

Let's consider the closed system in which charge moves harmonically and with constant acceleration. In this case the Hamilton function of the electron does not depend explicitly on time. Let's write it down as:

$$
H=K+U=E=\text { const },
$$

here $K, U$ are kinetic and potential energy and $E$ is a total energy of system. Then function of Lagrange is:

$$
L=K-U=2 K-E
$$

Let's write down classical action for the bounded electron:

$$
S=\int_{0}^{t} L \mathrm{~d} \tau=2 \int_{0}^{t} K \mathrm{~d} \tau-E t=S_{0}-E t
$$

but

$$
\Delta S=\int_{0}^{T_{1}} L_{1} \mathrm{~d} \tau-\int_{0}^{T_{2}} L_{2} \mathrm{~d} \tau=0
$$

where $T_{1}$ and $T_{2}$ are the periods of movement of the electron in system on the first and second orbit respectively. Then, considering the equation of Hamilton-Jacobi, for two different orbits 1 and 2 we have

$$
\Delta S=S^{2}-S^{1}=2 \int_{0}^{T_{2}} K^{2} \mathrm{~d} \tau-2 \int_{0}^{T_{1}} K^{1} \mathrm{~d} \tau-\left(E_{2} T_{2}-E_{1} T_{1}\right)=0
$$

However (see statements 1 and 2, mentioned above)

$$
\left(E_{2} T_{2}-E_{1} T_{1}\right)=h v T_{p h}=h
$$

is action for a emitted/absorbed photon. Thus

$$
2 \int_{0}^{T_{2}} K_{2} \mathrm{~d} t-2 \int_{0}^{T_{1}} K_{1} \mathrm{~d} t=h
$$


that actually represents the first axiom of Bohr-Sommerfeld (1).

Let us consider for example an electron in the central field in the nonrelativistic limit. We have: $K=\frac{1}{2} p \dot{q}$ and $d t=\left(\frac{d q}{\dot{q}}\right)$, where $p=-\left(\frac{\partial H}{\partial \dot{q}}\right)$. Then expression (7) gives

$$
\oint p_{2} d q_{2}-\oint p_{1} d q_{1}=h
$$

which for s-state of atom of hydrogen gives a known ratio

$$
m r_{2}^{2} \dot{\varphi}_{2}-m r_{1}^{2} \dot{\varphi}_{1}=\hbar \text {. }
$$

Or, the same

$$
M_{2}-M_{1}=\hbar,
$$

where $M_{2}$ and $M_{1}$ are the angular momenta. To write down the last expression we used the fact that the obtained values $m r^{2} \dot{\varphi}$ formally coincides with the angular momenta for the electron in the coulomb field.

Let's put $M_{0}=0$ (that corresponds to $r_{0}=0$ ). In this case we have $M_{1}=M_{0}+\Delta M$, but $\Delta M=\hbar$, so we obtain

$$
M_{1}=M_{0}+\hbar=\hbar, M_{2}=M_{1}+\hbar=2 \hbar \ldots, M_{n}=n \hbar
$$

From this expression and a principle of mechanical similarity for the central potentials of $U \sim r^{k}$, we have

$$
\frac{M^{\prime}}{M}=\left(\frac{r^{\prime}}{r}\right)^{1+\frac{\kappa}{2}} ; \frac{E^{\prime}}{E}=\left(\frac{r^{\prime}}{r}\right)^{k}
$$

from where it follows:

$$
r_{n}=r_{1}(n)^{\frac{1}{1+\frac{\kappa}{2}}} \text { and } E_{n}=E_{1}(n)^{\frac{k}{1+\frac{\kappa}{2}}}
$$

Then for a classical harmonic oscillator $(k=2)$ from (11) we get:

$$
r_{n}=r_{1} \sqrt{n} ; E_{n}=E_{1} n
$$

and for atom of hydrogen

$$
r_{n}=r_{1} n^{2} ; \quad E_{n}=\frac{E_{1}}{n^{2}}
$$

The value $E_{1}$ in the last expression can be found easily from expression (6) $\left(E_{2} T_{2}-E_{1} T_{1}\right)=h$.

Accepting classical value of the period

$$
T=\pi e^{2} \sqrt{\frac{m}{2|E|^{3}}}
$$

and taking into account (13) $E_{2}=E_{1} / 4$ we have:

$$
E_{1}=\frac{m e^{4}}{2 \hbar^{2}}
$$

Thus we showed that so-called quantization of system (axioms of Bohr-Sommerfeld) arises in absolutely classical way from the intrinsic properties of the electromagnetic field and cannot be treated as quantum property of space or matter.

\section{Harmonic Oscillator}

There is a common misconception that the addition term of $1 / 2$, which appears in the energy of the harmonic oscillator, is a quantum effect and is associated with the so-called zero-oscillations. Due to the methodological 
importance of this question, we discuss it here in a little more detail in the non-relativistic limit, and show that it is a purely classical effect.

Accordingly to classical mechanics, the energy of the harmonic oscillator is:

$$
E=m\left(\dot{r}^{2}+\omega^{2} r^{2}\right) / 2
$$

Considering that for the harmonic oscillator average value $\bar{T}=\bar{U}$, we obtain for the average energy for the period:

$$
E_{n}=m r_{n}^{2} \omega^{2}
$$

To carry out transition from an initial state of system to the final one $E_{n} \rightarrow E_{k}$, we should "take away" energy from our oscillator by electromagnetic field.

It is known that emission of an electromagnetic field by a moving charge differ from zero only if we integrate for the full period $T$ of movement in the course of which the emission or absorption appears. It corresponds to the fact that the full photon instead of a part is emitted/absorbed, that is the generated field satisfying to a periodicity condition.

The factor of proportionality between energy and frequency for a free electromagnetic field is $\hbar$ :

$$
\Delta E=E_{n}-E_{k}=\hbar \omega_{n k}
$$

(Once again we emphasize here that as it follows from Einstein's and Debye works, the constant $\hbar$ concerns only to the electromagnetic field and do not appears in any way from matter properties, or the size of the system under consideration). Expression (12) gives a ratio between energy levels, however from Equation (18) it is clear that the residual energy $E_{0}=U\left(r_{1}\right)$ cannot be emitted by a photon with energy $\hbar \omega$, because

$$
\Delta E=E_{1}-E_{0}=m r_{1}^{2} \omega^{2}-m r_{1}^{2} \omega^{2} / 2=E_{1} / 2<\hbar \omega
$$

Therefore this additive constant (which appears owing to the shape of the potential) should be simply added to the expression (12):

$$
E_{n}=n E_{1}+E_{1} / 2=\hbar \omega(n+1 / 2) .
$$

Thus, the additive constant $1 / 2$ appears naturally from classical consideration.

\section{Quantum Mechanics Is the Fourier-Transformed Classical Mechanics}

In standard textbooks of quantum mechanics problems arise and are solved for isolated systems, when the free/bounded electromagnetic field is not included in the Hamiltonian of the system. For example a harmonic oscillator, the hydrogen atom, molecular potentials, etc. Thus on the one hand any changes in the system (transitions between levels) are associated with the photons, but on the other hand, this electromagnetic field does not appear in such Hamiltonian. Reasonable questions arise: where the electromagnetic field is and why it does not appears in the Hamiltonian $H$ ? How these electromagnetic fields are taken into account for the quantization of such a systems?

At the beginning of the 20-th century the equations describing the quantum system have been intuitively guessed and accepted for the calculations (despite the emerging issues). It became possible because their predicted results were perfectly consistent with the experiments at the time. However, the meaning of the wave equations and the wave function itself is still not completely understood. In this section we will show sense of the formalism of quantum mechanics making a start from bases of classical mechanics

For simplicity, consider the one-dimensional motion. The generalization to three dimensions is obvious. Suppose we have the classical equation for energy of system:

$$
H=E
$$

Here $H$-is its classical Hamilton function and $E$-is the total energy of the system. Let's consider a particle in the field of $U(x)$. For a total energy of system we have two possibilities:

1) $E<0$ the system is bounded, we have a periodic movement,

2) $E>0$ the system is unbounded, we have a free movement.

Any function (and the Hamilton one in particular) can be expanded in a Fourier series $(E<0)$ or integral $(E>$ 
0) in the complete set of functions. Photons in turn can be described by harmonic waves which form such complete set of functions for the expansion we interested in:

$$
\varphi=\exp \left(-i k_{\alpha} x^{\alpha}\right)
$$

where $k_{\alpha}$ and $x_{\alpha}$ are 4 -vectors.

Consider $E<0$, that corresponds to a discrete spectrum in quantum mechanics. The case of continuous spectrum, when $E>0$, differs only by replacement of sums by integrals, but the entire derivation of the equations is done similarly.

Let's apply to Equation (21) the Fourier-transform on coordinate $x$ :

$$
\int H(k, x) \varphi(k, x) \mathrm{d} x=\int E \varphi(k, x) \mathrm{d} x,
$$

or

$$
\int \frac{p^{2}}{2 m} \mathrm{e}^{-\frac{i}{\hbar}(p x-E t)} \mathrm{d} x+\int U(x) \mathrm{e}^{-\frac{i}{\hbar}(p x-E t)} \mathrm{d} x=\int E \mathrm{e}^{-\frac{i}{\hbar}(p x-E t)} \mathrm{d} x
$$

from where we obtain:

$$
\int \mathrm{d} x\left[-\frac{\hbar^{2}}{2 m} \frac{\partial^{2}}{\partial x^{2}}+U(x)=-i \hbar \frac{\partial}{\partial t}\right] \mathrm{e}^{-\frac{i}{\hbar}(p x-E t)},
$$

or

$$
\int \mathrm{d} x[(\hat{H}-E) \varphi=0]
$$

where $\hat{H}$ is the Hamilton operator of the system under consideration.

We note here that the replacement of an electron by a positron (formally changes the sign in the exponent for opposite one), leads to the replacement of $t$ by $-t$ in Equation (25). In Equation (26) in the brackets we have the Hamilton operator $\hat{H}$, which actually is the Liouville operator (it corresponds to electron in potential $U(x)$, without the coupled electromagnetic field), so it has a complete set of orthogonal eigenfunctions.

Let $\Psi_{k}(x)$ be a complete set of eigenfunctions of the operator $\hat{H}$, then we can write down

$$
\varphi(p, x)=\sum_{m} a_{m}(p) \Psi_{m}(x),
$$

and the Equation (26) becomes

$$
\int \mathrm{d} x \sum_{m} a_{m}(p)\left[(\hat{H}-E) \Psi_{m}=0\right]
$$

or in non-relativistic limit one can write:

$$
\hat{H} \Psi_{m}(x)=E_{m} \Psi_{m}(x) .
$$

This is the equation of Schrödinger in coordinate representation. It is clear that if in Equation (23) we integrate on $p$ instead of coordinate, in the same way we obtain the Schrödinger equation, but now in $p$-representation.

$$
\hat{H} \Psi_{m}(p)=E_{m} \Psi_{m}(p) .
$$

Let's make now inverse transformation of expression (28). We have:

$$
\iint \mathrm{d} x \sum_{m} \varphi^{*}(k, x) a_{m}\left[\hat{H} \Psi_{m}-E \Psi_{m}\right] \mathrm{d} p=0
$$

considering that

$$
\varphi^{*}(k, x)=\sum_{n} a_{n}^{*}(p) \Psi_{n}^{*}(x)
$$

we can obtain 


$$
\iint \mathrm{d} x \mathrm{~d} p \sum_{m} \sum_{n} a_{m} a_{n}^{*} \Psi_{n}^{*}(x)[\hat{H}-E] \Psi_{m}(x)=0
$$

or in another form:

$$
\int \mathrm{d} p \sum_{m} \sum_{n} a_{m} a_{n}^{*}\left\langle\Psi_{n}|[\hat{H}-E]| \Psi_{m}\right\rangle=0
$$

That immediately implies matrix notation of quantum mechanics.

So, we have shown that:

- The quantum mechanics is the Fourier-transformed classical mechanics, and transformation goes on the function of the electromagnetic field which does not appear obviously in the Schrödinger equations, remaining out of consideration framework.

- The quantum theory is an incomplete (local) theory because it is based on an incomplete (local) Equation (29) (of Schrödinger) instead of the complete (non-local) Equation (28) where the electromagnetic field appears as expansion coefficients $a_{m}(p)$ under summation and integration.

- So-called wave functions are not "probability density" but are just eigenfunctions of the operator of Liouville which forms the problem of Sturm-Liouville for incomplete system. And these are the eigenfunctions that allow us to make decomposition of the bounded electromagnetic field coupled with a charge to include it into consideration. It should be stressed here, the theory based on the Equation (28) does not suffer of the wave function collapse problem, and the Einstein-Podolsky-Rosen paradox does not appears. In consequence with the expression (28), the wave function is defined completely by the photon, and its collapse appears within the wavelength size of the photon (integration on $d x$ in the expression (28)). So, within this complete theory there do not appear movements characterized by velocities more than light speed, as it take place in the Einstein-Podolsky-Rosen paradox for the Schrödinger Equation (29).

To conclude, the uncertainty principle $\Delta p \Delta x \sim \hbar$ should be mentioned briefly. As it was discussed above, any measurement occurs with the assistance of a photon. In this way, we can measure the coordinates of the object with the precision of up to $\Delta x=\lambda / \cos \varphi$ where $\lambda$ is wavelength of the photon. However in the course of coordinate measurement the photon transfers a part of their impulse to the measured object so we can write $\Delta p=\hbar k \cos \varphi$. Combining the first expression with the second one we have $\Delta p \Delta x \sim \hbar$. On the other hand, in view of that the phase is an invariant, we can conclude that symmetric expression also take place $\Delta E \Delta t \sim \hbar$.

\section{Adiabatic Invariant}

From astronomical observations it is well established that we live in a non-stationary Universe, in which all parameters change over time. By taking into account this fact, let's consider an isolated mechanical system making finite movement. Without loss of a generality we consider only one coordinate $q$, characterizing movement of the system. Suppose also that movement of the system is characterized by a certain parameter $r$. Here we can take $r=r_{u}$-radius of the Universe or $r=R$-scalar curvature of space. The final result will not depend on our choice.

Let the parameter $r$ adiabatically change with time, i.e.

$$
T \ll r / \dot{r}
$$

where $T$-is the characteristic time, or period of motion of our system. From this relation one can obtain estimation for the proper frequency of the system satisfying the adiabatic condition:

$$
v \gg 10^{-18}\left[\mathrm{~s}^{-1}\right]
$$

which actually corresponds to the always fulfilled relation $\lambda_{p h} \ll r_{u}$ (the wavelength of a photon is much less than the size of the Universe). It is clear that the system in question (photon) in this case is not isolated, and for the total system energy we have the linear relationship $\dot{E} \sim \dot{r}$. The Hamiltonian of the system, in turn, depends on parameter $r$, therefore

$$
\dot{E}=\frac{\partial H}{\partial t}=\frac{\partial H}{\partial r} \frac{\partial r}{\partial t} .
$$

Averaging this expression on the period, we obtain 


$$
\oint\left(\frac{\partial p}{\partial E} \frac{\overline{\partial E}}{\partial t}+\frac{\partial p}{\partial r} \frac{\partial r}{\partial t}\right) d q=0,
$$

or designating our adiabatic invariant by $h$, get from this expression

$$
\overline{\partial h} / \partial t=0,
$$

where

$$
h=\oint p d q / 2 \pi
$$

is the Planck's constant on their sense. Considering that

$$
2 \pi \frac{\partial h}{\partial E}=\oint \frac{\partial p}{\partial E} d q=T
$$

we can write down the energy of a photon as

$$
E=h v+E_{0}
$$

It should be noted here, that the integration constant $E_{0} \neq 0$ for general case.

\section{Relation between the Geometry of the Universe and the Value of Planck Constant}

Earlier we have shown how the quantum mechanical picture of surrounding reality appears. In the present section we obtain the important quantitative characteristic of the quantum theory_value of the Planck constant, from observable geometry of the Universe.

It is well known that General Relativity formulated on Riemann manifold has some difficulties. Among the most significant the following should be mentioned:

- The presence of singularities.

- Inability to take into account the "large numbers" of Eddington-Dirac which formally suggest a relation between cosmological and the quantum values.

- The cosmological constant which has no explanation within the framework of GR.

To search for a solution of these problems we must consider more general extensions of the Riemann geometry. One of its possible natural extensions is the geometry of Riemann-Cartan in which the theory of EinsteinCartan with asymmetrical connections can be developed. There is a variety of reasons for such a choice:

1) The theory of Einstein-Cartan satisfies the principle of relativity and also the equivalence principle and does not contradict the observational data.

2) It follows necessarily from gauge theory of gravitation.

3) It is free from the problem of singularities.

4) It suggests the most natural way to explain the cosmological constant as a non-Riemannian part of the scalar curvature of space, caused by torsion.

Within Riemann's geometry, as it is known, for the tensor of electromagnetic field we have relation:

$$
A_{v ; \mu}-A_{\mu ; v}=A_{\gamma, \mu}-A_{\mu, v}
$$

(Due to the symmetry of connections, the covariant derivatives of 4-potencial in the field tensor can be substituted by partial derivatives). But in the case of Einstein-Cartan theory with asymmetrical connections, the relation (42) is not more fulfilled and an additional term in the tensor of electromagnetic field appears.

To construct a theory we need the Lagrangian, which includes a natural linear invariant-the scalar curvature obtained by reduction of the Riemann-Cartan tensor of curvature. Let's accept from the beginning that curvature of space is small (that conforms to experiment) and, therefore, we can neglect by quadratic invariants in Lagrangian, having written down action for a gravitational field and a matter in Riemann-Cartan geometry this manner:

$$
S=S_{g}+S_{m}=\frac{c^{3}}{16 \pi G} \int_{\Omega} \tilde{R} \sqrt{-g} \mathrm{~d} \Omega+\frac{1}{c} \int_{\Omega} \tilde{\mathcal{L}}_{m} \sqrt{-g} \mathrm{~d} \Omega
$$


Here $c$-light velocity, $G$-gravitational constant, g-determinant of the metric tensor $g^{\alpha \beta}, \tilde{R}$ is scalar curvature and $\tilde{\mathcal{L}}_{m}$ is the Lagrangian of the matter which have been written down for Riemann-Cartan manifold, $d \Omega=d^{4} x$. Varying it we obtain

$$
\delta S_{g}=-\frac{c^{3}}{16 \pi G} \int_{\Omega}\left(\tilde{R}_{\alpha \beta}-\frac{1}{2} g_{\alpha \beta} \tilde{R}\right) \delta g^{\alpha \beta} \sqrt{-g} \mathrm{~d} \Omega
$$

and

$$
\delta S_{m}=\frac{1}{2 c} \int_{\Omega} \tilde{T}_{\alpha \beta} \delta g^{\alpha \beta} \sqrt{-g} d \Omega,
$$

or

$$
\tilde{R}_{\alpha \beta}-\frac{1}{2} g_{\alpha \beta} \tilde{R}=\frac{8 \pi G}{c^{4}} \tilde{T}_{\alpha \beta}
$$

Here $\tilde{T}_{\alpha \beta}$ is a tensor of density of energy-momentum of the matter in geometry of R-C. Simplifying on indexes we have:

$$
\tilde{R}=-\frac{8 \pi G}{c^{4}} \tilde{T}
$$

or in other form

$$
(R-4 \Lambda)=-\frac{8 \pi G}{c^{4}} \tilde{T}
$$

where $R$ —is the scalar formed of the Riemann's tensor, $\Lambda=(R-\tilde{R}) / 4$ and $\tilde{T}$-trace of tensor $\tilde{T}_{\alpha \beta}$ of electromagnetic field in R-C geometry.

In the right side of Equation (47) we have the value associated with the difference of geometry from the Riemann one (the trace of a tensor $T_{\alpha \beta}$ for the electromagnetic field is equal to zero in Riemann geometry because of symmetry of connections) that we want to evaluate. The problem of the direct estimation of the value of $\tilde{T}$ is that we do not know the true metric of the Universe we live in. We also do not know the real connection coefficients of our space. For this reason, we cannot directly calculate the value that we are interested in. Accordingly, we cannot just write out a corresponding amendment to the energy of electromagnetic field. However we can estimate this value indirectly, considering that the left part of expression (47) contains observable values. As it follows from the section "adiabatic invariant" for the action of electromagnetic field we have:

$$
S=S_{0}-h
$$

where $S_{0}$ is an constant of integration and $h$-is the adiabatic invariant (Planck constant) caused by slowly changing curvature of space in the Riemann-Cartan Universe. Then, considering that the trace of the tensor $T_{\alpha \beta}$ for the electromagnetic field is equal to zero in Riemann's geometry, we can write at once from (47)

$$
(R-4 \Lambda) \frac{c^{4}}{8 \pi G} \approx 2 \frac{h}{\Delta t_{0}}=2 h v_{0}
$$

We emphasize here that on the left side of this expression, we have the observed quantities which characterize the Universe geometry, while on the right side, appears the Planck constant, which in turn, characterize a microcosm. The value $\Delta t_{0}$ is minimum possible interval of time corresponding to action $h$. To find it we notice that energy of the corresponding electromagnetic field can change only by the value $h v$. (see first part of the paper). Let's consider as an example the atom of hydrogen (for our purposes we could consider any system). The first Bohr orbit is characterized by value $M_{1}=m_{e} a_{0} V_{0}=\hbar$, where $m_{e}$ is the electron mass, $a_{0}$-Bohr radius and $V_{0}$-velocity of the electron at first Bohr orbit. State with $M_{0}=0$ is not achievable for our system. As radius reduces from $a_{0}$ to the Compton wavelength $\lambda_{c} / 2 \pi$, the value $M_{1}=\hbar$ cannot be changed, for the photon cannot be emitted. So we can write $\lambda_{c} c / 2 \pi=a_{0} V_{0}$, or $v_{0}=c / a_{0}=2 \pi V_{0} / \lambda_{c}=5.6652 \times 10^{18}\left[\mathrm{~s}^{-1}\right]$. Here we need to emphasize especially: time, as well as space, are continuous, i.e. they do not quantized. The interval $\Delta t_{0}=1.7651 \times 10^{-19}[\mathrm{~s}]$ is the minimum interval of time, corresponding to value $h$. From expression (49), we 
can write

$$
(R-4 \Lambda) \frac{c^{3} a_{0}}{16 \pi G} \approx h
$$

where

$$
R=4 \pi^{2} \frac{H_{0}^{2}}{c^{2}}
$$

Let's estimate the Planck constant. The measured values of the Hubble constant were presented in paper [7] $H_{0}=74.2 \pm 3.6\left[\mathrm{kms}^{-1} \mathrm{Mpc}^{-1}\right]$ and paper [8] $H_{0}=73.8 \pm 2.4\left[\mathrm{kms}^{-1} \mathrm{Mpc}^{-1}\right]$. Let's take for our assessment average value $H_{0}=74\left[\mathrm{kms}^{-1} \mathrm{Mpc}^{-1}\right]$. Cosmological constant $\Lambda$ we adopt according to measurements $\Omega_{\Lambda}=0.7$ and we accept critical density $\rho_{c}=1.88 \times 10^{-29}\left[\mathrm{~g} \mathrm{~cm}^{-3}\right]$. Then, from expression (50) we obtain value for the Planck's constant $h=6.6 \times 10^{-27}[\mathrm{erg} s]$, that coincides to within the second sign with experimental value.

Recently, the issue of a possible change of the fine structure constant $\alpha$ on time is widely debated, so for convenience, we put here another interesting relationship, which follows from (50)

$$
(R-4 \Lambda) \frac{c^{4}}{16 \pi G} \approx 2 \pi m_{e} c^{2} \alpha
$$

\section{Other Observational Effects}

The results suggested in present work can be proved by independent experiments. The most basic of them is of course the double slit experiment. Recently it was accurately carried out by [9], which clearly argued for our model of non-local quantum theory. Another possible experiment could be a measurement of the blackbody spectrum in far Reyleigh-Jeans region. As it was shown earlier, if the geometry of Riemann-Cartan has non-zero scalar curvature, in expression for energy of electromagnetic field appears the additional term $h v$. The energy of one photon in this case is:

$$
E_{v}=E_{v}^{0}+h v
$$

where $v$ is a frequency of a photon, and a small additional energy

$$
E_{v}^{0}=\frac{1}{16 \pi} \int\left(E^{2}+H^{2}\right) \mathrm{d} V-h v
$$

Integration here is carried out over the volume of one photon. Intensity of the black body emission in this case one can write as

$$
B_{v}=\left(E_{v}^{0}+h v\right) \frac{2 v^{2}}{c^{2}} \frac{1}{\exp \left\{\frac{E_{v}^{0}+h v}{\kappa T}\right\}-1}
$$

As one can see, in Wien and in close Reyleigh-Jeans regions the spectrum is almost coincide with Planck one because of small value of $E_{v}^{0}$. However it is clear that the small additive energy $E_{v}^{0}$ can lead to some deviations from Planck spectrum in far Reyleigh-Jeans region and, probably, such deviation could be measured experimentally. It is necessary to emphasize that such experiment has independent great importance because will allow to state an assessment to the value $E_{v}^{0}$ and to throw light on the geometrical nature of electromagnetic field.

\section{Conclusions}

In present work we made the next logical step towards implementation of the program started by Einstein and Schrödinger in the fifties of the XX century (model of Einstein-Cartan-Schrödinger). Namely we show that the Planck constant is actually the adiabatic invariant of the electromagnetic field, characterized by scalar curvature of space of the Riemann-Cartan geometry. The main results of present work are: 
For the first time we obtained the ratio between Riemannian scalar curvature of the Universe, the Cosmological constant and Planck's constant (see expression (50)), true up to the second decimal place $\left(\Omega_{\Lambda} \gg \Omega_{b}\right)$.

It is stressed that due to change of geometry of the Universe, the Planck constant changes with time too.

The physical sense of the cosmological constant, as the no-Riemannian part of the scalar curvature, which appears due to the presence of torsion (asymmetrical connections), is revealed for the first time.

Dependence of the fine structure constant on the total scalar curvature of the Universe is obtained (52).

Within the used framework, natural unification of gravitation with the quantum theory is obtained.

The spectral density of the blackbody radiation is obtained in linear on curvature invariants approach.

Bases of the quantum theory are reconsidered and the physical sense of wave function is found. It is shown that if we eliminate an unnatural axiom of existence of wave function of matter, the huge discrepancy between calculated by the QFT methods and observed cosmological constant, disappears.

The approach based on the Equation (28), completely removes a problem of collapse of wave function and classically resolves the Einstein-Podolsky-Rosen paradox. According to expression (28) "wave function", as it should be, is completely determined by corresponding electromagnetic field and its collapse occurs at scales of wavelength of the photon (integration on $d x$ in expression (28)). Thus in this way there is no need for transmission of a signal with a superluminal speed as it takes place in paradox of E-P-R for the Schrödinger equation (29).

\section{Acknowledgements}

I would like to acknowledge Dr. J. Saucedo for the valuable criticism and comments. I also grateful to the Pulkovo Observatory team and particularly to Dr. E. Poliakow for the opportunity to spent part of 2008 year in the Observatory.

\section{References}

[1] Jammer, M. (1967) The Conceptual Development of Quantum Mechanics. Mc Graw Hill, New York.

[2] Ljjas, A., Steinhardt, P.J. and Loeb, A. (2013) Inflationary Paradigm in Trouble after Planck 2013. http://xxx.lanl.gov/abs/1304.2785

[3] Einstein, A. (1906) Zur Theorie der Lichterzengung und Lichtabsorption. Annales der Physik, 199-206. http://dx.doi.org/10.1002/andp.19063250613

[4] Einstein, A. (1905) Uber einen die Erzeugung und Verwandlung des Lichtes betreffenden heuristischen Gesichtspunkt. Annalen der Physik, 132-148. http://dx.doi.org/10.1002/andp.19053220607

[5] Debye, P. (1910) Der Wahrscheinfichkeitsbegriff in der Theorie der Strahlung. Annalen der Physik, 1427-1434. http://dx.doi.org/10.1002/andp.19103381617

[6] Grangier, P., Roger, G. and Aspect, A. (1986) Experimental Evidence for a Photon Anticorrelation Effect on a Beam Splitter: A New Light on Single-Photon Interferences. Europhys. Lett., 1, 173-179. http://dx.doi.org/10.1209/0295-5075/1/4/004

[7] Riess, A.G., Macri, L., Casertano, S., et al. (2009) A Redetermination of the Hubble Constant with the Hubble Space Telescope from a Differential Distance Ladder. Ap. J., 699, 539-563. http://dx.doi.org/10.1088/0004-637X/699/1/539

[8] Riess, A.G., Macri, L., Casertano, S., et al. (2011) A 3\% Solution: Determination of the Hubble Constant with the Hubble Space Telescope and Wide Field Camera 3. Ap.J., 730, 119-136. http://dx.doi.org/10.1088/0004-637X/730/2/119

[9] Demjanov, V.V. (2010) Experiments Performed in Order to Reveal Fundamental Differences between the Diffraction and Interference of Waves and Electrons. arXiv: 1002.3880. 